\title{
Highly Efficient, Low-Cost, and Magnetically Recoverable FePt-Ag Nanocatalysts: Towards Green Reduction of Organic Dyes
}

\author{
Yang Liu ${ }^{1,2,+}$, Yuanyuan Zhang ${ }^{1,2}$, Qiangwei Kou ${ }^{1,2}$, Yue Chen ${ }^{1,2}$, Yantao Sun ${ }^{2}$, \\ Donglai Han ${ }^{3,+}$, Dandan Wang ${ }^{4}$, Ziyang Lu ${ }^{5}$, Lei Chen ${ }^{1,2}$, Jinghai Yang ${ }^{1,2, *}$ and \\ Scott Guozhong Xing ${ }^{6, *}$ \\ 1 College of Physics, Jilin Normal University, Siping 136000, China; liuyang@jlnu.edu.cn (Y.L.); \\ 13944139606@163.com (Y.Z.); 13944949603@163.com (Q.K.); 17649973053@163.com (Y.C.); \\ chenlei@jlnu.edu.cn (L.C.) \\ 2 Key Laboratory of Functional Materials Physics and Chemistry of the Ministry of Education, \\ Jilin Normal University, Changchun 130103, China; syt@jlnu.edu.cn \\ 3 School of Materials Science and Engineering, Changchun University of Science and Technology, \\ Changchun 130022, China; dlhan_1015@cust.edu.cn \\ 4 Technology Development Department, GLOBALFOUNDRIES (Singapore) Pte. Ltd., 60 Woodlands \\ Industrial Park D, Street 2, Singapore 738406, Singapore; DANDAN.WANG@globalfoundries.com \\ 5 School of Environment and Safety Engineering, Jiangsu University, Zhenjiang 212013, China; \\ lzy@mail.ujs.edu.cn \\ 6 United Microelect Corp. Ltd., 3 Pasir Ris Dr 12, Singapore 519528, Singapore \\ * Correspondence: jhyang1@jlnu.edu.cn (J.Y.); Scott_Xing@UMC.com or guozhongupenn@gmail.com (S.G.X.); \\ Tel./Fax: +86-434-329-4566 (J.Y. \& S.G.X.) \\ + These authors contributed equally to this work.
}

Received: 1 April 2018; Accepted: 11 May 2018; Published: 14 May 2018

\begin{abstract}
Nowadays, synthetic organic dyes and pigments discharged from numerous industries are causing unprecedentedly severe water environmental pollution, and conventional water treatment processes are hindered due to the corresponding sophisticated aromatic structures, hydrophilic nature, and high stability against light, temperature, etc. Herein, we report an efficient fabrication strategy to develop a new type of highly efficient, low-cost, and magnetically recoverable nanocatalyst, i.e., FePt-Ag nanocomposites, for the reduction of methyl orange (MO) and rhodamine B (RhB), by a facile seed deposition process. X-ray diffraction results elaborate that the as-synthesized FePt-Ag nanocomposites are pure disordered face-centered cubic phase. Transmission electron microscopy studies demonstrate that the amount of Ag seeds deposited onto the surfaces of FePt nanocrystals increases when increasing the additive amount of silver colloids. The linear correlation of the MO and $\mathrm{RhB}$ concentration versus reaction time catalyzed by FePt-Ag nanocatalysts is in line with pseudo-first-order kinetics. The reduction rate constants of $\mathrm{MO}$ and RhB increase with the increase of the amount of Ag seeds. FePt-Ag nanocomposites show good separation ability and reusability, and could be repeatedly applied for nearly complete reduction of $\mathrm{MO}$ and $\mathrm{RhB}$ for at least six successive cycles. Such cost-effective and recyclable nanocatalysts provide a new material family for use in environmental protection applications.
\end{abstract}

Keywords: FePt-Ag nanocomposites; magnetic properties; catalytic reduction; organic dyes

\section{Introduction}

At present, water environmental pollution has become a major global issue. Excessive emissions of the dye effluents derived from the textile, leather, food, paper, plastic, rubber, and pharmaceutical 
industries are one of the most important water pollution sources [1-4]. These dispersed dye molecules block sunlight from reaching the lower depths of the water, reduce the dissolved oxygen level in the water, and thereby inhibit the growth and development of marine plants and animals [5-7]. Most seriously, most of these dyes are mutagenic and carcinogenic and cause severe damage to human life [8-10]. Therefore, it is necessary to remove the dyes from wastewater for environmental protection and remediation. Unfortunately, it is hard to treat organic dye contaminants because their structures are stable and biologically nondegradable [11,12].

Conventional treatment techniques to remove and decolorize organic dyes include adsorption, biodegradation, ultrafiltration, photochemical, and electrochemical methods [13]. Due to the constraints like the complex aromatic structure and recalcitrant nature of the dyes or the high operating cost, the above techniques are difficult to popularize in practical applications. Recently, photocatalytic degradation in the presence of semiconductor photocatalysts (e.g., typically, $\mathrm{TiO}_{2}$ and $\mathrm{ZnO}$ ) and catalytic reduction of noble metal catalysts (e.g., typically, $\mathrm{Ag}$ and $\mathrm{Au}$ ) have developed as alternatives to conventional water treatment methods for the treatment of organic dyes [14-16]. For instance, $\mathrm{Li}$ et al. synthesized $\mathrm{TiO}_{2}$ to catalyze and degrade methyl orange (MO) under UV light irradiation [17]. However, even though the photocatalytic method is cost effective, the photocatalytic efficiency is low and not effective enough for the dye degradation. Another disadvantage is that the electrons and the holes of the semiconductor photocatalysts can only be excited under ultraviolet light irradiation.

Most recently, the catalytic reduction method for the removal of dyes by using noble metal nanocatalysts has been regarded as a competitive technology because of the high efficiency and simplicity of its design and operation. It must be mentioned that the noble metal nanocatalysts encounter an obstacle because they are difficult to efficiently separate and recycle after the catalytic process by traditional methods such as centrifugation or filtration. To address this problem, many researchers have tried to immobilize the nanocatalysts onto magnetic supporting materials [18-21]. Zhang et al. synthesized $\mathrm{Fe}_{3} \mathrm{O}_{4} / \mathrm{Ag}$ composites to catalyze rhodamine B (RhB) [22]. Bimetallic FePt nanocrystals have high chemical stability and fast magnetic responsiveness [23]. Interestingly, research results have found that FePt nanocrystals themselves have catalytic degradation abilities [24,25]. Therefore, considerable efforts have been devoted to the design and preparation of FePt-Ag nanocrystals for the degradation of dyes.

In this work, with the aim of ensuring the ability of the proposed catalytic degradation system not only for a specific dye but also for other industrial dyes, methyl orange (MO) and rhodamine B (RhB) were selected as representative model dye pollutants. Polyethyleneimine dithiocarbamate (PEI-DTC) was employed as a polymer to prepare FePt-Ag nanocomposites by the seed deposition process. By adjusting the additive amount of silver colloids or the additive amount of FePt nanocrystals, we control the amount of Ag seeds deposited on the modified surfaces of FePt nanocrystals. In addition, in order to investigate the catalytic ability of FePt-Ag nanocomposites, we investigate the effects of the amount of $\mathrm{Ag}$ seeds on the catalytic capacities of the catalytic reduction of $\mathrm{MO}$ and $\mathrm{RhB}$ with the help of sodium borohydride $\left(\mathrm{NaBH}_{4}\right)$. A schematic illustration of the synthesis and application of $\mathrm{FePt}-\mathrm{Ag}$ nanocomposites is shown in Figure 1. 


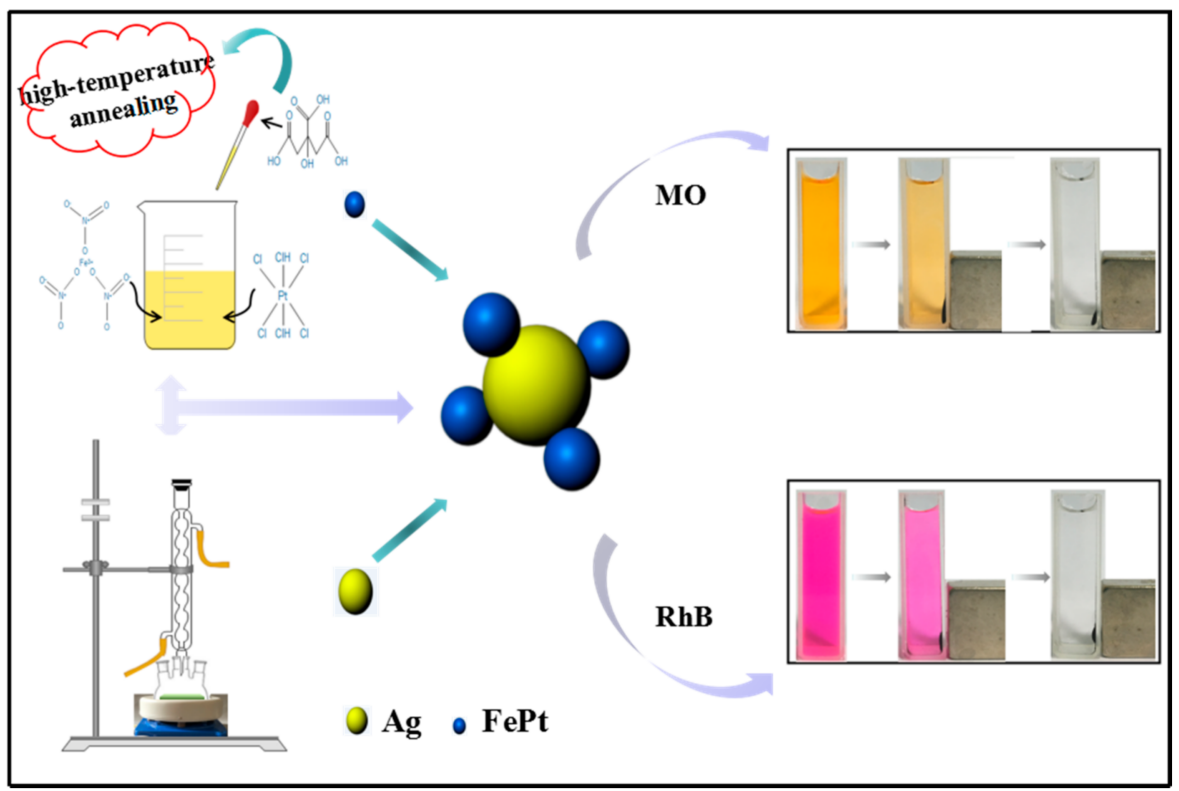

Figure 1. Schematic illustration of the synthesis and application of FePt-Ag nanocomposites.

\section{Experimental}

\subsection{Materials}

The initial materials included iron (III) nitrate nonahydrate $\left(\mathrm{Fe}\left(\mathrm{NO}_{3}\right)_{3} \cdot 9 \mathrm{H}_{2} \mathrm{O}\right)$, chloroplatinic acid hexahydrate $\left(\mathrm{H}_{2} \mathrm{PtCl}_{6} \cdot 6 \mathrm{H}_{2} \mathrm{O}\right)$, citric acid monohydrate $\left(\mathrm{C}_{6} \mathrm{H}_{8} \mathrm{O}_{7} \cdot \mathrm{H}_{2} \mathrm{O}\right)$, polyethyleneimine (PEI, branched, $\mathrm{MW} \approx 25,000 \mathrm{~g} / \mathrm{mol})$, potassium hydroxide $(\mathrm{KOH})$, carbon disulfide $\left(\mathrm{CS}_{2}\right)$, silver nitrate $\left(\mathrm{AgNO}_{3}\right)$, and trisodium citrate dihydrate $\left(\mathrm{C}_{6} \mathrm{H}_{5} \mathrm{Na}_{3} \mathrm{O}_{7} \cdot 2 \mathrm{H}_{2} \mathrm{O}\right)$. All chemical reagents in this work were used as received without further purification.

\subsection{Preparation of Silver Colloids}

Noble metal nanocrystals have received both fundamental and practical attention owing to their potential applications in many fields such as ultrasensitive biosensing, imaging agents, photothermal therapy, and in particular catalysts because of their unique and tunable optical properties and high catalytic activities [26-33]. In the present work, the silver colloids were prepared based on the work by Yang et al. [34]. Briefly, $36 \mathrm{mg}$ of $\mathrm{AgNO}_{3}$ was dissolved in $200 \mathrm{~mL}$ of deionized water under vigorous stirring at $90{ }^{\circ} \mathrm{C}$. The solution was heated to near boiling after $15 \mathrm{~min}$, and then $40 \mathrm{mg}$ of $4 \mathrm{~mL}$ $\mathrm{C}_{6} \mathrm{H}_{5} \mathrm{Na}_{3} \mathrm{O}_{7} \cdot 2 \mathrm{H}_{2} \mathrm{O}$ was injected into the solution drop by drop. The temperature of the solution was immediately reduced to $85^{\circ} \mathrm{C}$ and the mixture was stirred continuously for another $40 \mathrm{~min}$. Finally, the reaction mixture was allowed to cool down to room temperature and stored in the dark.

\subsection{Preparation of Ag@PEI-DTC Solution}

This process included two steps. The first step was the synthesis of PEI-DTC. Quantities of $50 \mathrm{mg}$ of PEI and $325 \mathrm{mg}$ of $\mathrm{KOH}$ were dissolved in $25 \mathrm{~mL}$ methanol. After the mixture solution was degassed, $347.5 \mu \mathrm{L}$ of $\mathrm{CS}_{2}$ was added dropwise to the mixture solution; PEI-DTC aqueous solution was thus obtained. The second step was the synthesis of Ag@PEI-DTC solution. A quantity of $60 \mathrm{~mL}$ of silver colloids was centrifuged and then dispersed in $20 \mathrm{~mL}$ of methanol. The obtained silver solution was added into the PEI-DTC solution drop by drop. After the reaction mixture was kept at room temperature for $1 \mathrm{~h}$, the resultant product was collected by centrifugation ( $5000 \mathrm{rpm}, 10 \mathrm{~min}$ ) and subsequently washed with deionized water and ethanol several times. Finally, the obtained Ag@PEI-DTC seeds were stored in $4 \mathrm{~mL}$ of deionized water. 


\subsection{Preparation of FePt Nanocrystals}

The FePt nanocrystals were synthesized using the sol-gel method. Details of the preparation have been published in our previous work [35]. In general, $234 \mathrm{mg}$ of $\mathrm{Fe}\left(\mathrm{NO}_{3}\right)_{3} \cdot 9 \mathrm{H}_{2} \mathrm{O}, 20 \mathrm{~mL}$ of $\mathrm{H}_{2} \mathrm{PtCl}_{6} \cdot 6 \mathrm{H}_{2} \mathrm{O}$, and $162 \mathrm{mg}$ of $\mathrm{C}_{6} \mathrm{H}_{8} \mathrm{O}_{7} \cdot \mathrm{H}_{2} \mathrm{O}$ were dissolved in $50 \mathrm{~mL}$ of deionized water with stirring to form the sol. The solution was polymerized to form a gel at $80^{\circ} \mathrm{C}$. FePt nanocrystals were successfully synthesized with precursors heat-treated in argon atmosphere for $2 \mathrm{~h}$ at $400{ }^{\circ} \mathrm{C}$ with a heating rate of $2{ }^{\circ} \mathrm{C} \min ^{-1}$.

\subsection{Preparation of FePt-Ag@PEI-DTC Nanocomposites}

Most recently, considerable efforts have been devoted to the design and preparation of multifunctional nanocomposites. Compared to the bulk materials, nanostructured materials possess large surface area while retaining their excellent electrical, optical, and magnetic properties [36-43]. A quantity of $10 \mathrm{mg}$ of FePt nanocrystals was dispersed in $4 \mathrm{~mL}$ of deionized water and then added dropwise into the obtained Ag@PEI-DTC solution under vortex. FePt-Ag@PEI-DTC nanocomposites were shaken for $1 \mathrm{~h}$ and then washed with deionized water and ethanol three times; the result was named FePt-Ag $10 \mathrm{mg}-60 \mathrm{~mL}$. The experiment was carried out again under the same conditions as FePt-Ag $10 \mathrm{mg}-60 \mathrm{~mL}$, but the additive amount of silver colloids was adjusted to 90 and $120 \mathrm{~mL}$, with the results named FePt-Ag $10 \mathrm{mg}-90 \mathrm{~mL}$ and FePt-Ag $10 \mathrm{mg}-120 \mathrm{~mL}$, respectively. In addition, in order to study the effect of the additive amount of FePt nanocrystals on the amount of Ag seeds deposited on the modified surfaces of FePt nanocrystals, the volume of the silver colloids was kept the same as in FePt-Ag $10 \mathrm{mg}-60 \mathrm{~mL}$, but the mass of FePt nanocrystals was changed to $5 \mathrm{mg}$; the result was named FePt-Ag $5 \mathrm{mg}-60 \mathrm{~mL}$.

\subsection{Application of FePt-Ag Nanocomposites for Catalytic Reduction of $M O$ and $R h B$}

The catalytic reduction of $\mathrm{MO}$ and $\mathrm{RhB}$ in the presence of $\mathrm{NaBH}_{4}$ was studied and monitored by a $\mathrm{UV}-\mathrm{vis}$ spectroscopy to explore the catalytic activity of FePt-Ag $10 \mathrm{mg}-120 \mathrm{~mL}$. The detailed reaction process was as follows: $1 \mathrm{mg}$ of FePt-Ag $10 \mathrm{mg}-120 \mathrm{~mL}$ was added to $2 \mathrm{~mL}$ of $\mathrm{MO}$ or RhB aqueous solution $\left(40 \mathrm{mg} \cdot \mathrm{L}^{-1}\right)$. Subsequently, $0.1 \mathrm{~mL}$ of $\mathrm{NaBH}_{4}$ aqueous solution $(0.1 \mathrm{M}$, freshly prepared each time before use) was injected into the mixture solution. UV-vis spectra of the solution were recorded every $6 \mathrm{~min}$. The first obtained data were designated as the values for the reaction time $t=0$. The rate constant of the reduction process was determined by measuring the change in absorption as a function of time. It could be observed that the solution color changed gradually from colored to colorless. In order to investigate the reusability of the prepared samples, the FePt-Ag $10 \mathrm{mg}-120 \mathrm{~mL}$ nanocatalysts were separated from the reaction solution using a magnet after the completion of the reduction process. The obtained magnetic nanocatalysts were washed with ethanol and deionized water three times and reused in the next reaction process. The same procedure for the catalytic reduction of each of $\mathrm{MO}$ and $\mathrm{RhB}$ was repeated six times.

\subsection{Characterization Methods}

Structure characterization of the as-synthesized samples was carried out on a Rigaku D/Max-2500 copper (Rigaku Corporation, Tokyo, Japan) rotating-anode X-ray diffractometer (XRD) using Cu $\mathrm{K} \alpha$ radiation $(40 \mathrm{kV}, 200 \mathrm{~mA})$. Ultraviolet-visible spectroscopy (UV-vis) absorbance spectra were recorded on a Shimadzu UV 3600 spectrophotometer (Shimadzu Corporation, Tokyo, Japan) in the range of 350-800 $\mathrm{nm}$. The detailed structure of the obtained samples was characterized using a JEOL JSM-7800F field emission scanning electron microscope (FESEM) (JEOL Ltd., Tokyo, Japan) at an acceleration voltage of $20 \mathrm{kV}$ and a JEOL 2100 transmission electron microscope (TEM) operating at $200 \mathrm{kV}$. The X-ray photoelectron spectrum was measured with a Thermo Scientific ESCALAB 250Xi X-ray photoelectron spectroscope (XPS) (Thermo Fisher Scientific, Waltham, MA, USA). Magnetic 
properties were measured using a Quantum Design MPMS3 superconducting quantum interference device (SQUID) magnetometer (Quantum Design, Inc., San Diego, CA, USA).

\section{Results and Discussion}

\subsection{Structure of FePt-Ag Nanocomposites}

The crystal structure of pure FePt and FePt-Ag nanocomposites was investigated using XRD. Figure $2 \mathrm{a}, \mathrm{b}$ present $\mathrm{XRD}$ patterns of the pure FePt nanocrystals and the FePt-Ag nanocomposites with different additive amounts of FePt nanocrystals (FePt-Ag $5 \mathrm{mg}-60 \mathrm{~mL}$ and FePt-Ag $10 \mathrm{mg}-60 \mathrm{~mL}$ ), and of the FePt-Ag nanocomposites with different additive amounts of silver colloids (FePt-Ag $10 \mathrm{mg}-60 \mathrm{~mL}$, FePt-Ag $10 \mathrm{mg}-90 \mathrm{~mL}$, and FePt-Ag $10 \mathrm{mg}-120 \mathrm{~mL}$ ), respectively. As for the pure FePt nanocrystals, three primary peaks of (111), (200), and (220) around $39.8^{\circ}, 46.4^{\circ}$, and $67.6^{\circ}$ are observed and no other trace of impurities are detected, indicating that the as-synthesized FePt nanocrystals are pure disordered face-centered cubic phase [44]. After silver colloids are added to FePt nanocrystals, three additional diffraction peaks at about $38.3^{\circ}, 44.5^{\circ}$, and $64.7^{\circ}$ appear; these can be well attributed to the (111), (200), and (220) of Ag (Joint Committee on Powder Diffraction Standards, JCPDS card no. 04-0783) [45], respectively. With the decrease of the additive amount of FePt nanocrystals or the increase of the additive amount of silver colloids, the intensity of Ag diffraction peaks is obviously enhanced, but the position of $\mathrm{Ag}$ diffraction peaks does not change significantly. On the basis of these XRD results, we preliminarily conclude that the number of Ag seeds attached on the FePt surfaces can be adjusted by changing the additive amount of silver colloids or FePt nanocrystals.
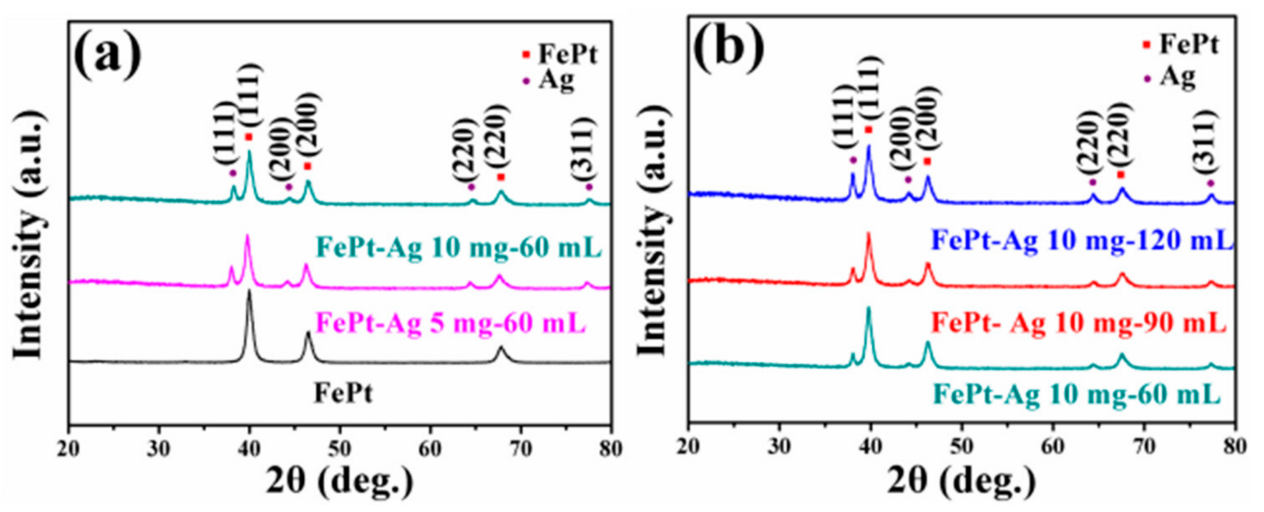

Figure 2. XRD patterns of the pure FePt nanocrystals and the FePt-Ag nanocomposites with different additive quantities of FePt nanocrystals (FePt-Ag $5 \mathrm{mg}-60 \mathrm{~mL}$ and FePt-Ag $10 \mathrm{mg}-60 \mathrm{~mL}$ ) (a), and of the FePt-Ag nanocomposites with different additive amounts of silver colloids (FePt-Ag $10 \mathrm{mg}-60 \mathrm{~mL}$, FePt-Ag 10 mg-90 mL, and FePt-Ag 10 mg-120 mL) (b).

UV-vis absorbance spectra were used to further confirm that the Ag seeds were firmly immobilized on the surfaces of FePt nanocrystals. Figure 3 shows the UV-vis absorbance spectra of silver colloids, FePt nanocrystals, and FePt-Ag $10 \mathrm{mg}-120 \mathrm{~mL}$ nanocomposites. It is well established that silver colloids exhibit a plasmon absorption band in the visible region on account of the coherent excitation of the free electrons within the conduction band [46]. In general, the plasmon absorption band of silver nanocrystals is around $420 \mathrm{~nm}$, while Fe and Pt nanocrystals have no specific absorption peak in this region [47]. As can be seen from Figure 3, the as-prepared silver colloids have a characteristic absorption peak at $430 \mathrm{~nm}$, but no such absorption peak is observed for the FePt nanocrystals. An interesting finding is that the absorption peak of FePt-Ag $10 \mathrm{mg}-120 \mathrm{~mL}$ shows a slight red shift from 430 to $435 \mathrm{~nm}$ compared with that of the silver colloids, which might be attributed to the polarizability of the metallic silver owing to the formation of the FePt-Ag nanocomposites [48]. 


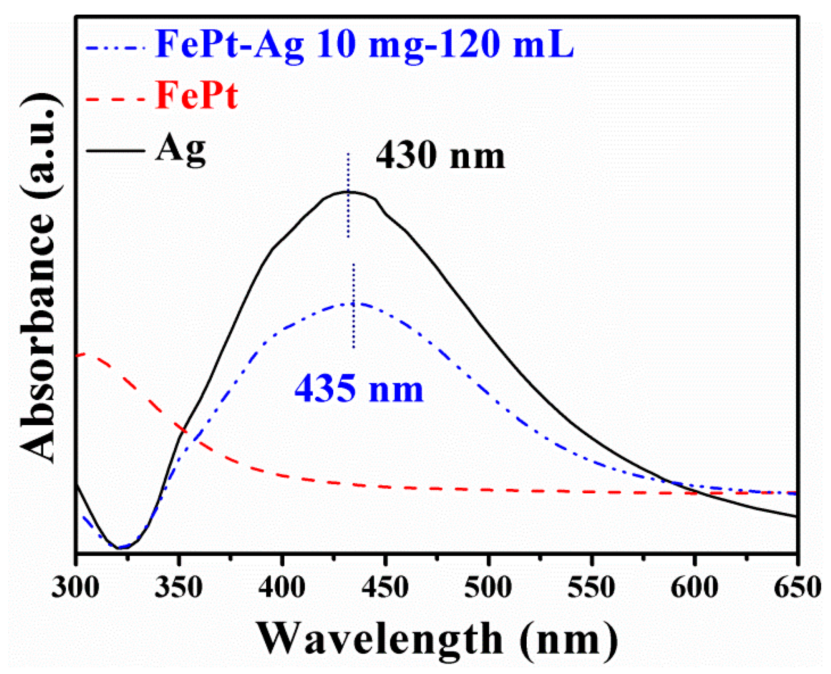

Figure 3. UV-vis absorbance spectra of silver colloids (black curve line), pure FePt nanocrystals (red dotted line), and FePt-Ag $10 \mathrm{mg}-120 \mathrm{~mL}$ nanocomposites (blue dotted line).

\subsection{Morphology of FePt-Ag Nanocomposites}

The morphology and detailed structure of the samples were investigated by SEM and TEM. In particular, the TEM technique is a direct and important methodology for structure analysis in the materials engineering technologies [49-61]. Figure 4 shows typical low-magnification TEM images of pure FePt nanocrystals, pure silver colloids, and FePt-Ag $5 \mathrm{mg}-60 \mathrm{~mL}$ and FePt-Ag $10 \mathrm{mg}-60 \mathrm{~mL}$ nanocomposites. The shapes of both the pure FePt nanocrystals and silver colloids are nearly spherical, and their average particle sizes are $11 \pm 1.5$ and $41 \pm 2 \mathrm{~nm}$, respectively. The corresponding particle size histogram of the pure FePt nanocrystals indicates that FePt nanocrystals have a narrow particle size distribution (Figure S1). The inset of Figure $4 \mathrm{a}$ is the corresponding high-resolution TEM (HRTEM) image, which shows the characteristic spacings of $0.223 \mathrm{~nm}$ for the (111) lattice planes of face-centered cubic FePt. From the TEM images of FePt-Ag 5 mg-60 mL and FePt-Ag 10 mg-60 mL, Ag seeds are found to be coherent with FePt nanocrystals owing to the effect of the two chelating sulfur groups provided by the PEI-DTC polymers. It is commonly known that the brightness in the TEM image reflects the ability of the transmitted electrons from the different substances and is proportional to the atomic number of the substances [62]. Unfortunately, because both FePt and $\mathrm{Ag}$ nanocrystals have high atomic numbers, it is hard to distinguish the two nanomaterials from TEM images. TEM images of the FePt nanocrystals with different addition quantities of the silver colloids (FePt-Ag $10 \mathrm{mg}-60 \mathrm{~mL}$, FePt-Ag $10 \mathrm{mg}-90 \mathrm{~mL}$, and FePt-Ag $10 \mathrm{mg}-120 \mathrm{~mL}$ ) are exhibited in Figure 5. In order to investigate the influence of the additive amount of silver colloids on the number of Ag seeds attached to the FePt surfaces, energy-dispersive spectroscopy (EDS) was performed to determine the composition of the nanostructures, as shown in Figure S2. The EDS results confirm that FePt-Ag nanocomposites are composed of $\mathrm{Fe}, \mathrm{Pt}$, and $\mathrm{Ag}$, and that the amount of $\mathrm{Ag}$ seeds adhered to the surfaces of $\mathrm{FePt}$ nanocrystals increases with the increase in the additive amount of silver colloids. This is consistent with the observation from XRD results. The Si peak could be assigned to the Si substrates for loading the samples. HRTEM images show that the interplanar spacings of 0.223 and $0.235 \mathrm{~nm}$ match well with those of the (111) planes of FePt and Ag, respectively. The selected area electron diffraction (SAED) pattern of FePt-Ag $10 \mathrm{mg}-120 \mathrm{~mL}$ is presented in the inset of Figure 5d. The bright and discrete diffraction spots can be assigned to the diffractions of either FePt nanocrystals or Ag seeds. HRTEM and SAED results further testify the coexistence of FePt and Ag. 


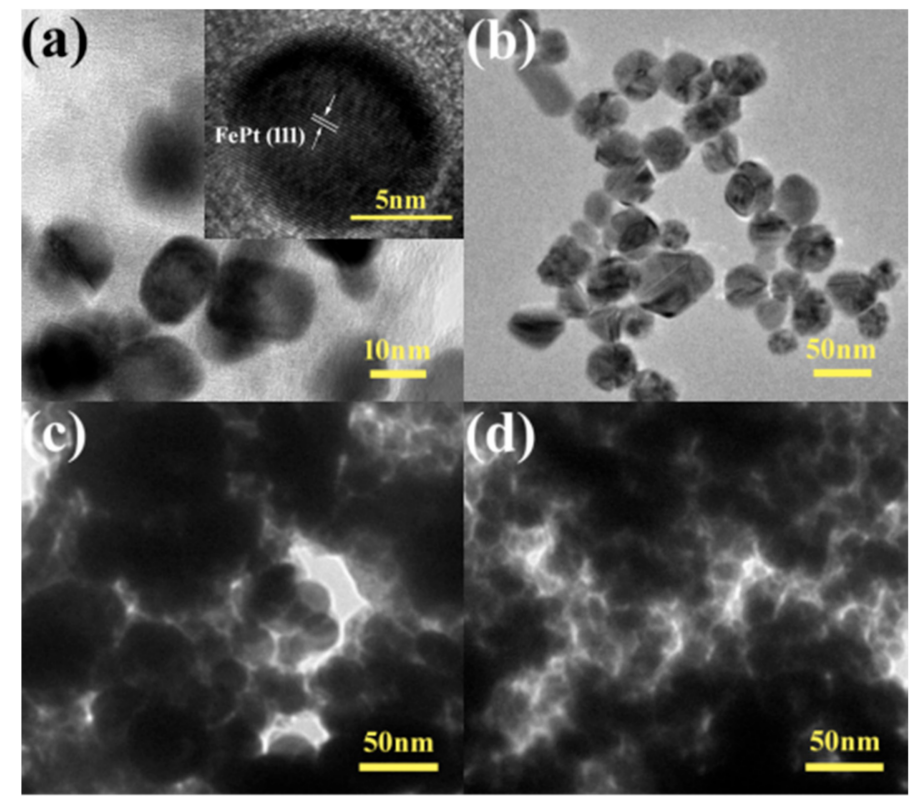

Figure 4. TEM images of the pure FePt nanocrystals with high-resolution TEM (HRTEM) image (inset) (a), pure silver colloids (b), and FePt-Ag $5 \mathrm{mg}-60 \mathrm{~mL}$ (c) and FePt-Ag $10 \mathrm{mg}-60 \mathrm{~mL}$ nanocomposites (d).

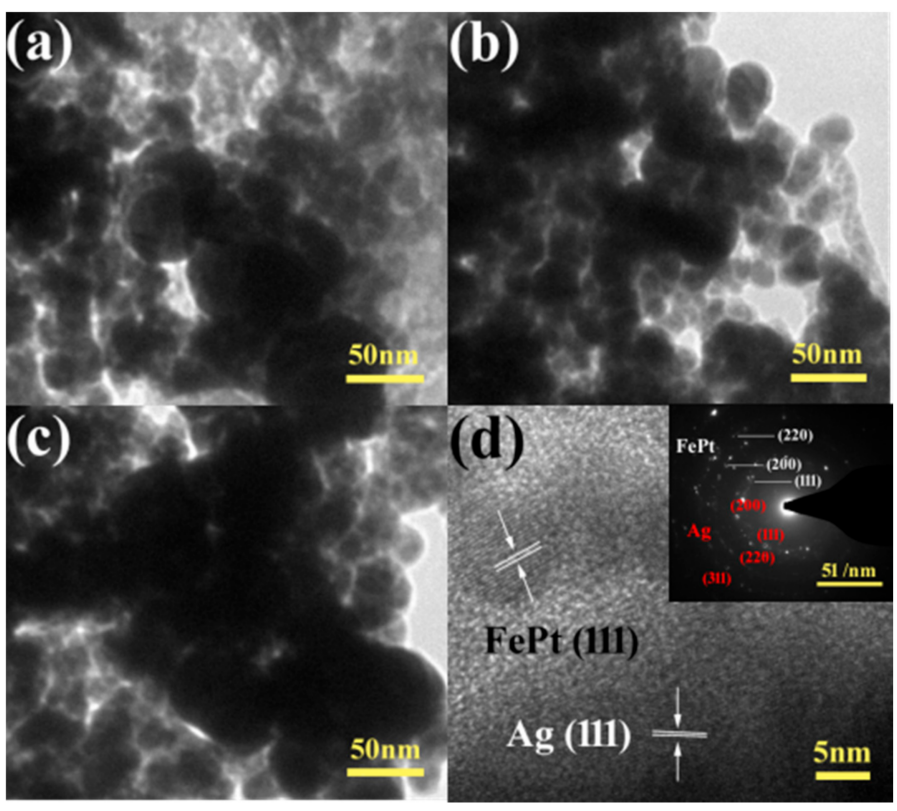

Figure 5. TEM images of FePt-Ag 10 mg-60 mL (a), FePt-Ag 10 mg-90 mL (b), and FePt-Ag $10 \mathrm{mg}-120 \mathrm{~mL}$ (c); (d) shows the corresponding selected area electron diffraction (SAED) pattern (inset) and HRTEM image of (c).

\subsection{XPS Studies of FePt-Ag Nanocomposites}

XPS measurements were carried out to characterize the chemical state of $\mathrm{Fe}, \mathrm{Pt}$, and $\mathrm{Ag}$ elements. High-resolution scans of $\mathrm{Fe} 2 p, \mathrm{Pt} 4 f$, and $\mathrm{Ag} 3 d$ for pure FePt nanocrystals and for FePt-Ag $10 \mathrm{mg}-60 \mathrm{~mL}$, FePt-Ag $10 \mathrm{mg}-90 \mathrm{~mL}$, and FePt-Ag $10 \mathrm{mg}-120 \mathrm{~mL}$ nanocomposites are shown in Figure 6. As for the pure FePt nanocrystals, the Fe $2 p$ spectrum shows two contributions- $2 p_{3 / 2}$ and $2 p_{1 / 2}$-resulting from the spin-orbit splitting, respectively located at 710.6 and $724.1 \mathrm{eV}$. The XPS spectrum of Pt exhibits double peaks with binding energies of 71.1 and $74.2 \mathrm{eV}$, corresponding to the 
standard peaks of pure $\mathrm{Pt} 4 f_{7 / 2}$ and $\mathrm{Pt} 4 f_{5 / 2}[63,64]$. The binding energy of FePt-Ag $10 \mathrm{mg}-60 \mathrm{~mL}$ is located at 367.4 and $373.4 \mathrm{eV}$, which can be assigned to $\mathrm{Ag} 3 d_{5 / 2}$ and $\mathrm{Ag} 3 d_{3 / 2}$, and matches well with the standard XPS spectrum of metallic Ag [53,54]. It should be pointed out that the intensity of Fe $2 p$ and $\mathrm{Pt} 4 f$ decreases and the intensity of $\mathrm{Ag} 3 d$ increases with the increase of the additive amount of the silver colloids, since the intensity of XPS spectra is proportional to the atomic concentration [65]. Another interesting point is that the positions of $\mathrm{Fe} 2 p$ and $\mathrm{Pt} 4 f$ peaks shift toward the lower binding energy side, and the positions of Ag $3 d$ peaks shift toward the higher binding energy side. The shift of the binding energy provides evidence of an electronic exchange interaction between FePt and $\mathrm{Ag}$ [66]. Furthermore, in addition to $\mathrm{Fe}, \mathrm{Pt}, \mathrm{Ag}, \mathrm{O}$, and $\mathrm{C}$ peaks, no impurities are observed from the XPS survey scan spectra in Figure S3.
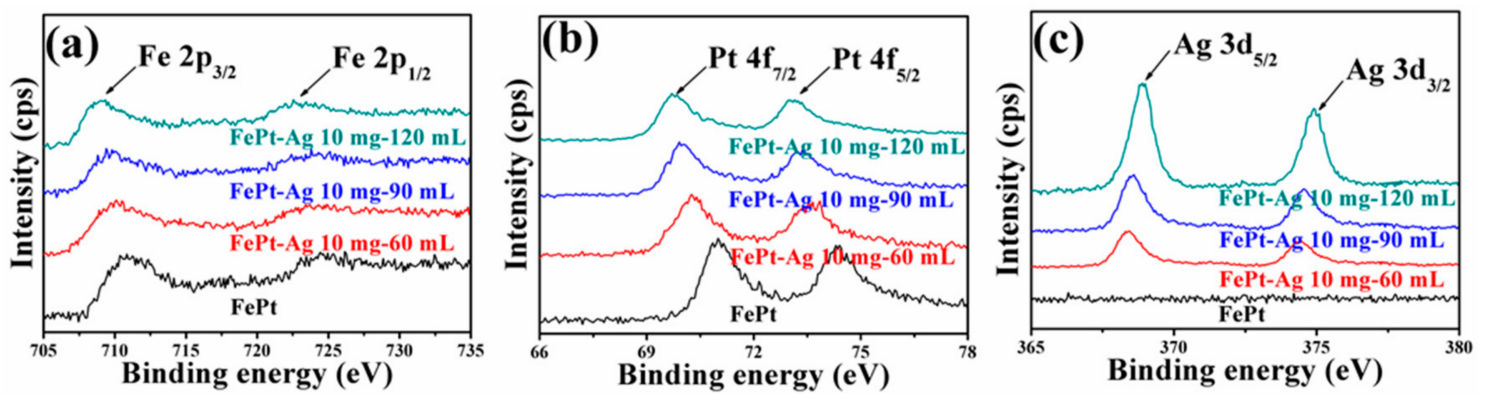

Figure 6. High-resolution XPS scans of Fe $2 p(\mathbf{a}), \mathrm{Pt} 4 f(\mathbf{b})$. and $\mathrm{Ag} 3 d$ (c) for pure FePt nanocrystals and for FePt-Ag 10 mg-60 mL, FePt-Ag 10 mg-90 mL, and FePt-Ag 10 mg-120 mL nanocomposites.

\subsection{Magnetism of FePt-Ag Nanocomposites}

The magnetic properties of the pure FePt nanocrystals and the FePt-Ag nanocomposites were characterized by SQUID at room temperature. Figure 7 shows the magnetic hysteresis $(M-H)$ loops of the pure FePt nanocrystals and the FePt-Ag nanocomposites. The magnetic saturation $(\mathrm{Ms})$ of the pure FePt nanocrystals is $4.8 \mathrm{emu} \cdot \mathrm{g}^{-1}$. It is obvious that the $M s$ value decreases with the decrease of the addition quantities of FePt nanocrystals or the increase of the additive amount of the silver colloids. On the basis of the previous XRD, TEM, EDS, and XPS results, both the decrease of the addition quantities of FePt nanocrystals and the increase of the additive amount of the silver colloids may lead to the increase of $\mathrm{Ag}$ seeds in FePt-Ag nanocomposites. Therefore, the decrease in $\mathrm{Ms}$ values may be ascribed to the increase in weight ratio of Ag seeds to FePt nanocrystals [67] or the diamagnetic contribution of the intact Ag seeds [68]. It must also be mentioned that although Ms values decrease due to the introduction of $\mathrm{Ag}$ seeds, the FePt-Ag nanocomposites still have strong magnetic responsivity and can be easily magnetically separated from aqueous solution by using an external magnet (as shown in Figure S4), which is beneficial to their economic applications and reuse.

The temperature-dependent magnetization $(M-T)$ of pure FePt and FePt-Ag $10 \mathrm{mg}-120 \mathrm{~mL}$ was measured under zero-field-cooled (ZFC) and 1000 Oe field-cooled (FC) conditions (Figure S5). The shape of ZFC-FC curves for the two samples is the typical form for weakly dipole-dipole interacting systems. The magnetization of FC curves monotonically increases with the decrease of temperature. The ZFC curves reach maximum at 86 and $81 \mathrm{~K}$ for the pure FePt and FePt-Ag $10 \mathrm{mg}-120 \mathrm{~mL}$, respectively, corresponding to the blocking temperature $\left(T_{B}\right)$. In general, the $T_{B}$ value is closely related to the nanocrystal size and the magnetic interaction between the nanocrystals [69]. It is reasonable to exclude the contribution of the FePt and Ag particle size to the variation of $\mathrm{T}_{\mathrm{B}}$ value. Thus, the $\mathrm{Ag}$ seeds in the FePt-Ag nanocomposites weaken the magnetic dipole-dipole interaction between adjacent FePt nanocrystals, and result in decrease of the $\mathrm{T}_{\mathrm{B}}$ value [70]. 

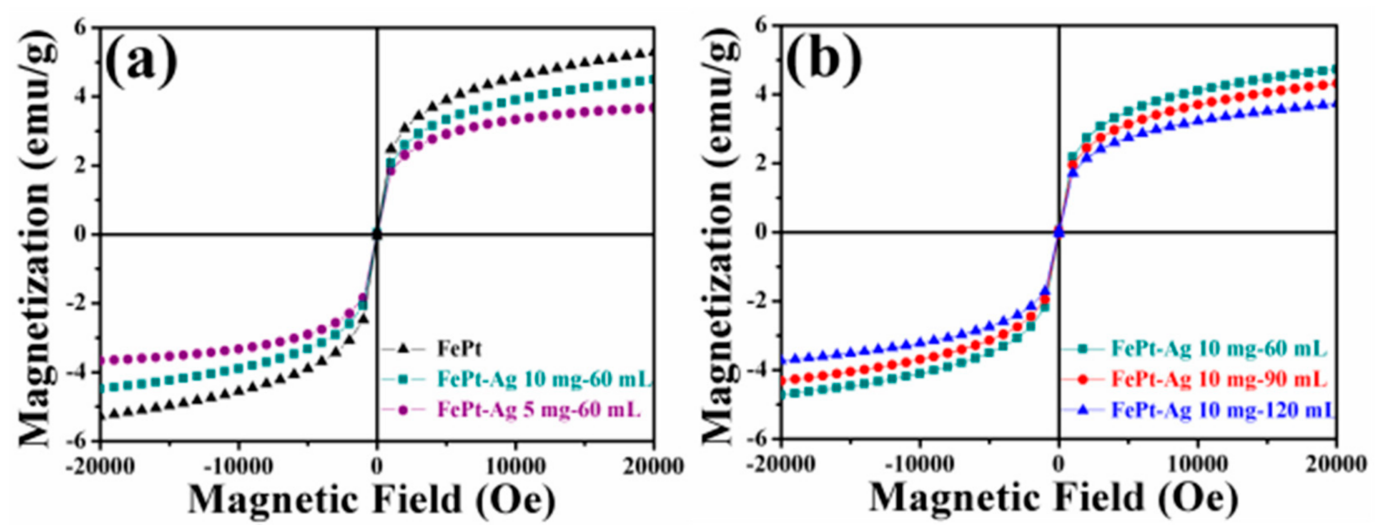

Figure 7. Magnetic hysteresis $(M-H)$ loops of the pure FePt nanocrystals and the FePt-Ag nanocomposites with different additive quantities of FePt nanocrystals (FePt-Ag $5 \mathrm{mg}-60 \mathrm{~mL}$ and FePt-Ag $10 \mathrm{mg}-60 \mathrm{~mL}$ ) (a), and the FePt-Ag nanocomposites with different additive amounts of silver colloids (FePt-Ag $10 \mathrm{mg}-60 \mathrm{~mL}$, FePt-Ag $10 \mathrm{mg}-90 \mathrm{~mL}$, and FePt-Ag $10 \mathrm{mg}-120 \mathrm{~mL}$ ) (b).

\subsection{Catalytic Studies of FePt-Ag Nanocomposites}

Previous works indicated that Ag nanocrystals played significant roles in the catalytic reduction of dye pollutants [71,72]. In order to study the catalytic performance of FePt-Ag nanocomposites on organic dyes, $\mathrm{MO}$ and $\mathrm{RhB}$ were selected as model dye pollutants. Figure 8 shows UV-vis absorption spectra of $\mathrm{MO}$ aqueous solution over time catalyzed by the different $\mathrm{FePt}-\mathrm{Ag}$ nanocomposites (FePt-Ag $10 \mathrm{mg}-60 \mathrm{~mL}$, FePt-Ag $10 \mathrm{mg}-90 \mathrm{~mL}$ and FePt-Ag $10 \mathrm{mg}-120 \mathrm{~mL}$ ) in the presence of $\mathrm{NaBH}_{4}$. As can be seen, the characteristic absorption peak of $\mathrm{MO}$ at $465 \mathrm{~nm}$ disappears within 30, 24, and $18 \mathrm{~min}$ of reaction time, respectively. This result indicates that the catalytic reduction and the complete removal of MO have been successfully achieved due to the inclusion of the FePt-Ag nanocomposites, which can be confirmed by the decoloration of $\mathrm{MO}$ in the inset of Figure 1 . The corresponding $\ln \left(C / C_{0}\right)$ versus reaction time plots are presented in Figure $8 \mathrm{~b}, \mathrm{~d}$,f. The concentration of MO at reaction time $t$ is denoted by $C$, and the initial concentration of $\mathrm{MO}$ at $t=0$ is regarded as $C_{0}$. The concentration of $\mathrm{NaBH}_{4}$ can be considered as a constant during the reaction period, because it is much more excessive relative to that of MO. The ratio of $C$ to $C_{0}$ is obtained from the relative intensity of respective absorbance $\left(A_{t} / A_{0}\right)$ at $465 \mathrm{~nm}$. The linear relationship of $\ln \left(C / C_{0}\right)$ versus reaction time shows that the reduction of $\mathrm{MO}$ by the $\mathrm{FePt}-\mathrm{Ag}$ nanocomposites matches well with pseudo-first-order kinetics [73]. The rate constant is determined by a linear plot of $\ln \left(C / C_{0}\right)$ versus reaction time. The correlation coefficients $R^{2}$ of the two samples are higher than 0.98 . The rate constants of $\mathrm{MO}$ are $0.14 \mathrm{~min}^{-1}, 0.18 \mathrm{~min}^{-1}$, and $0.24 \mathrm{~min}^{-1}$ using FePt-Ag $10 \mathrm{mg}-60 \mathrm{~mL}$, FePt-Ag $10 \mathrm{mg}-90 \mathrm{~mL}$, and FePt-Ag $10 \mathrm{mg}-120 \mathrm{~mL}$ as nanocatalysts, respectively. It is obvious that the rate constants for $\mathrm{MO}$ reduction increase with the increase of the additive amount of silver colloids. $\mathrm{NaBH}_{4}$ acts as the electron donor $\left(\mathrm{BH}_{4}{ }^{-}\right.$ions) and hydrogen supplier in this reaction [74]. When the FePt-Ag nanocomposites are added into the above reaction, the $\mathrm{BH}_{4}{ }^{-}$ions and the $\mathrm{MO}$ molecules are absorbed on the surfaces of the $\mathrm{FePt}-\mathrm{Ag}$ nanocomposites. The FePt-Ag nanocomposites can transfer the electrons from the donor $\mathrm{BH}_{4}{ }^{-}$to the acceptor $\mathrm{MO}$ molecules and the $\mathrm{BH}_{4}{ }^{-}$ions also transfer surface hydrogen to $\mathrm{MO}$ molecules. It is reasonable that if the noble metal nanomaterial loading is increased, the mass fraction of the noble metal nanomaterials is higher in the final samples, and, thus, a better catalytic performance should be accomplished [75]. 

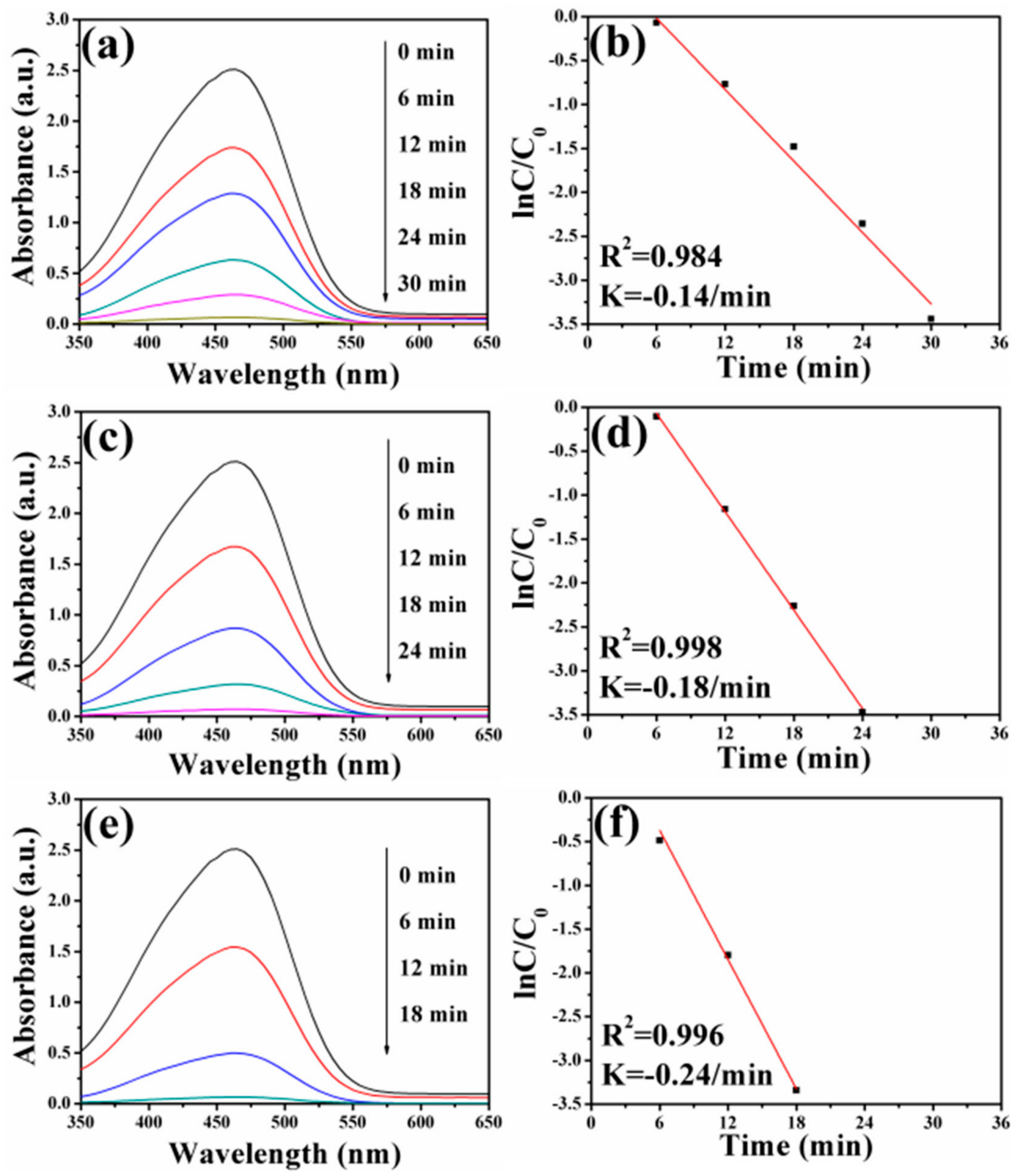

Figure 8. UV-vis absorption spectra of $\mathrm{MO}$ aqueous solution after reduction catalyzed by FePt-Ag 10 mg-60 mL (a), FePt-Ag 10 mg-90 mL (c), and FePt-Ag 10 mg-120 mL (e). (b,d,f) show the corresponding $\ln \left(C / C_{0}\right)$ versus reaction time plots.

In the case of $\mathrm{RhB}$, a similar decolorization from pink to transparent is also observed in the insets of Figure 1. The changes in UV-vis absorption spectra of RhB aqueous solution over time catalyzed by the different FePt-Ag nanocomposites are shown in Figure 9. The intensity of the maximum absorption peak at $555 \mathrm{~nm}$ for RhB gradually decreases. In addition, an identical trend that the rate constants for $\mathrm{RhB}$ reduction increase when increasing the additive amount of silver colloids is found. It indicates that the catalytic ability of as-prepared FePt-Ag nanocomposites is not only efficient to a specific dye but also to different industrial dyes. Notably, as can be seen from Figures S6 and S7, no appreciable changes of the UV-vis absorption spectra for $\mathrm{MO}$ and $\mathrm{RhB}$ are observed when FePt nanocrystals or $\mathrm{NaBH}_{4}$ only are chosen as the catalysts, which suggests that $\mathrm{MO}$ and $\mathrm{RhB}$ could not be effectively reduced in the presence of FePt or $\mathrm{NaBH}_{4}$ only. Therefore, the catalytic reaction ability against dye pollutants mainly depends on the presence and the number of Ag seeds in the catalysts. 

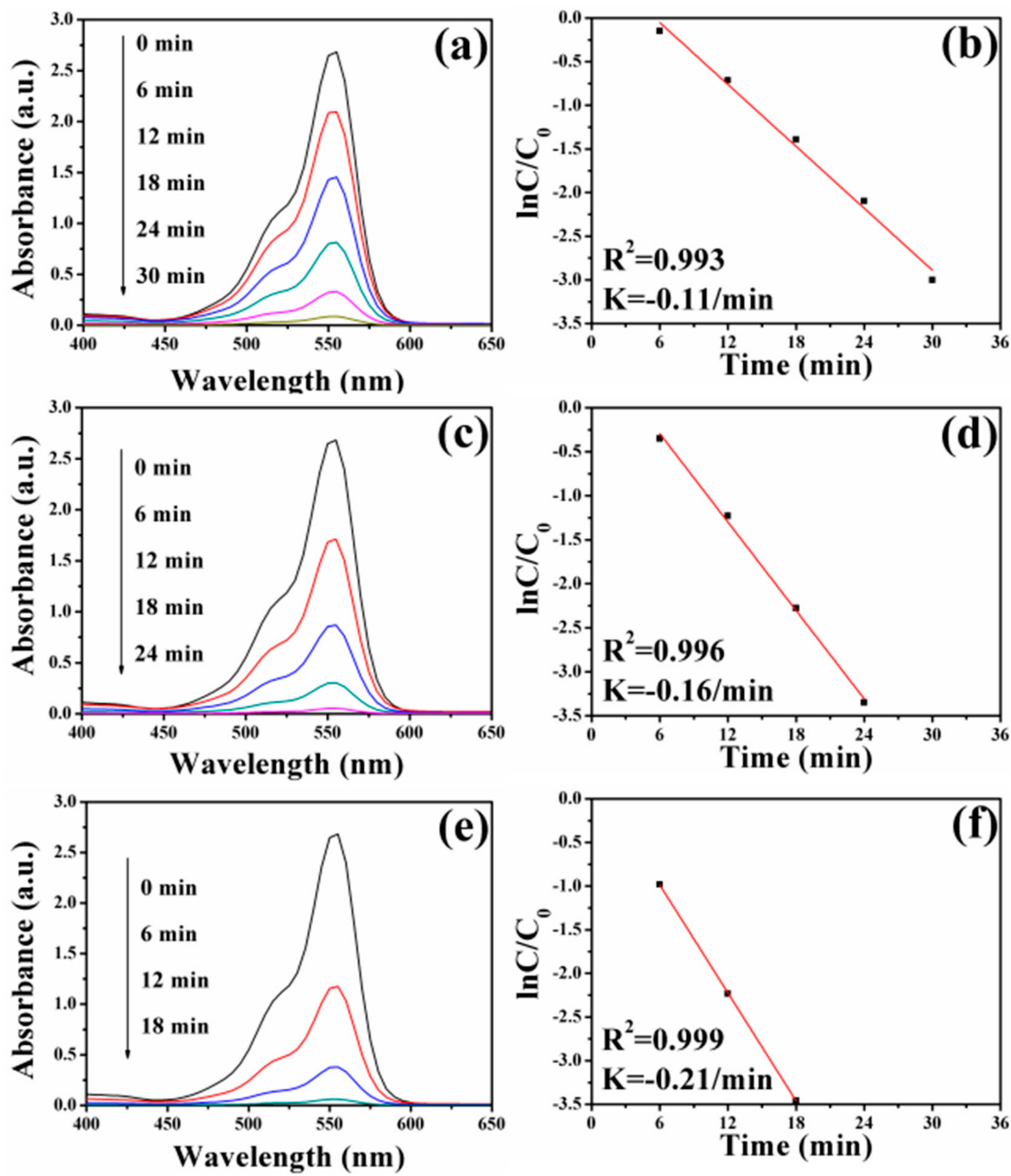

Figure 9. UV-vis absorption spectra of $\mathrm{RhB}$ aqueous solution after reduction catalyzed by FePt-Ag 10 mg-60 mL (a), FePt-Ag 10 mg-90 mL (c), and FePt-Ag 10 mg-120 mL (e). (b,d,f) show the corresponding $\ln \left(C / C_{0}\right)$ versus reaction time plots.

In order to investigate the recyclability of the FePt-Ag nanocomposites, FePt-Ag $10 \mathrm{mg}-120 \mathrm{~mL}$ was selected to be separated magnetically and reused after catalytic reduction. As shown in Figure 10, FePt-Ag $10 \mathrm{mg}-120 \mathrm{~mL}$ can be successfully recycled and reused against the two dye pollutants for at least six reaction runs with stable efficiency of around $100 \%$, which indicates that the FePt- $\mathrm{Ag}$ nanocomposites have excellent reusability and can serve as recoverable and efficient catalysts for dye pollutants. 

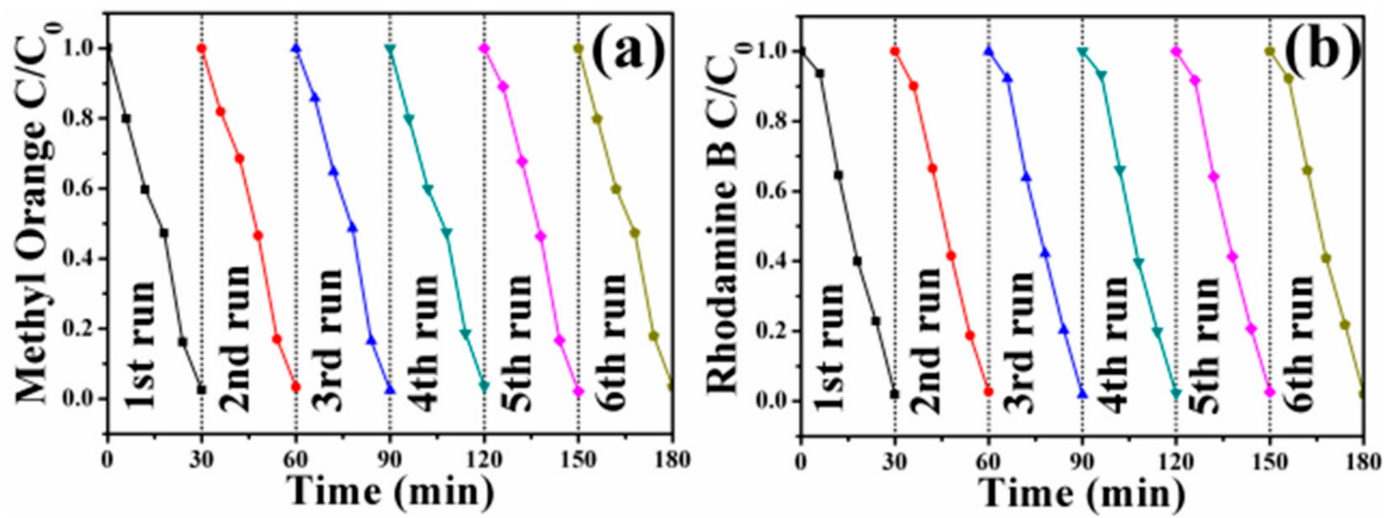

Figure 10. Six cycles of the removal of $\mathrm{MO}(\mathbf{a})$ and $\mathrm{RhB}(\mathbf{b})$ with FePt-Ag $10 \mathrm{mg}-120 \mathrm{~mL}$.

\section{Conclusions}

In summary, we have demonstrated the fabrication of pure FePt nanocrystals. Then, FePt-Ag nanocomposites were successfully synthesized through a novel seed deposition process. XRD, TEM, and SEM results indicate that the additive amount of silver colloids and FePt nanocrystals is essential to controlling the number of Ag seeds deposited on the PEI-DTC-modified surfaces of FePt nanocrystals. The increase of the number of Ag seeds attached to the FePt nanocrystals surfaces results in the shifts of Fe $2 p$ peaks and $\mathrm{Pt} 4 f$ peaks toward the lower binding energy side and shifts of $\mathrm{Ag} 3 d$ peaks toward the higher binding energy side. Although the Ms value decreases with the increase of the amount of Ag seeds attached on the FePt surfaces, the samples still have strong magnetic responsivity and can be easily separated from aqueous solution by applying a magnetic field. The pseudo-first-order kinetics are used to calculate the rate constant of $\mathrm{MO}$ and $\mathrm{RhB}$. The catalytic reaction ability of FePt-Ag nanocomposites remains almost consistent even after at least six cycles, demonstrating that the FePt-Ag nanocomposites can serve as recyclable nanocatalysts for organic dyes. This system could potentially be applied as highly efficient nanocatalysts to different types of organic dyes.

Supplementary Materials: Supplementary materials can be found at http:/ / www.mdpi.com/2079-4991/8/5/ 329/s1. Figure S1: Particle size histogram of the pure FePt nanocrystal. Inset shows the corresponding TEM images of the pure FePt nanocrystals. Figure S2: Energy-dispersive spectroscopy (EDS) spectra of FePt (a), FePt-Ag 10 mg-60 mL (b), FePt-Ag 10 mg-90 mL (c), and FePt-Ag 10 mg-120 mL (d). Figure S3: XPS survey scan spectra of pure FePt nanocrystals and FePt-Ag $10 \mathrm{mg}-120 \mathrm{~mL}$ nanocomposites. Figure S4: Photographs of the colloidal silver solution, FePt nanocrystals dispersed in deionized water, and FePt-Ag nanocomposites dispersed in deionized water and after gentle shaking. Figure S5: ZFC and FC curves of pure FePt nanocrystals and FePt-Ag $10 \mathrm{mg}-120 \mathrm{~mL}$ nanocomposites under an applied field of 1000 Oe. Figure S6: UV-vis absorption spectra of MO aqueous solution (a) and RhB aqueous solution (b) after reduction catalyzed by pure FePt nanocrystals. Figure S7: UV-vis absorption spectra of $\mathrm{MO}(\mathrm{a})$ and $\mathrm{RhB}(\mathrm{b})$ reduced by $\mathrm{NaBH}_{4}$ only.

Author Contributions: Y.L. conceived the experiments and wrote the manuscript; Y.Z., Q.K. and Y.C. carried out the experiments and tested the data; Y.S. and D.W. assisted with magnetic analyses; D.H., Y.Z. polished the whole manuscript; Z.L. and L.C. assisted with the catalytic analyses; J.Y. and S.G.X. helped perform the analyses with constructive theoretical discussions.

Funding: This research was funded by the National Natural Science Foundation of China (Grant Numbers 21676115, 51609100, 61705020, 61675090 and 61575080), Program for the development of Science and Technology of Jilin province (Grant Numbers 20160101287JC and 20150519024JH), Guangdong Province Science and Technology Projects (Grant Numbers 2015A030310342 and 2015A010103004), and Technology of Education Department of Jilin Province (Grant Number JJKH20170374KJ).

Conflicts of Interest: The authors declare no conflict of interest.

\section{References}

1. Xie, Y.J.; Yan, B.; Xu, H.L.; Chen, J.; Liu, Q.X.; Deng, Y.H.; Zeng, H.B. Highly Regenerable Mussel-Inspired $\mathrm{Fe}_{3} \mathrm{O}_{4} @$ Polydopamine-Ag Core-Shell Microspheres as Catalyst and Adsorbent for Methylene Blue Removal. ACS Appl. Mater. Interfaces 2014, 6, 8845-8852. [CrossRef] [PubMed] 
2. Deka, P.; Hazarika, A.; Dekaa, R.C.; Bharali, P. Influence of $\mathrm{CuO}$ morphology on the enhanced catalytic degradation of methylene blue and methyl orange. RSC Adv. 2016, 6, 95292-95306. [CrossRef]

3. Xu, D.; Cheng, F.; Lu, Q.; Dai, P. Microwave Enhanced Catalytic Degradation of Methyl Orange inAqueous Solution over $\mathrm{CuO} / \mathrm{CeO}_{2}$ Catalyst in the Absence andPresence of $\mathrm{H}_{2} \mathrm{O}_{2}$. Ind. Eng. Chem. Res. 2014, 53, 2625-2632. [CrossRef]

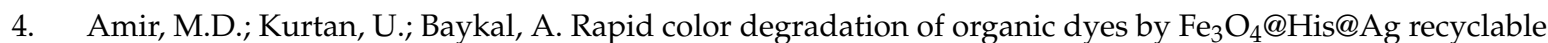
magnetic nanocatalyst. J. Ind. Eng. Chem. 2015, 27, 347-353. [CrossRef]

5. Saikia, P.; Miah, A.T.; Das, P.P. Highly efficient catalytic reductive degradation of various organic dyes by $\mathrm{Au} / \mathrm{CeO}_{2}-\mathrm{TiO}_{2}$ nano-hybrid. J. Chem. Sci. 2017, 129, 81-93. [CrossRef]

6. Soto-Quintero, A.; Romo-Uribe, Á.; Bermúdez-Morales, V.H.; Quijada-Garrido, I.; Guarrotxena, N. 3D-Hydrogel Based Polymeric Nanoreactors for Silver Nano-Antimicrobial Composites Generation. Nanomaterials 2017, 7, 209. [CrossRef] [PubMed]

7. Alshehri, S.M.; Almuqati, T.; Almuqati, N.; Al-Farraj, E.; Alhokbany, N.; Ahamad, T. Chitosan based polymer matrix with silver nanoparticles decorated multiwalled carbon nanotubes for catalytic reduction of 4-nitrophenol. Carbohydr. Polym. 2016, 151, 135-143. [CrossRef] [PubMed]

8. Li, X.G.; He, Y.Y.; Sui, H.; He, L. One-Step Fabrication of Dual Responsive Lignin Coated $\mathrm{Fe}_{3} \mathrm{O}_{4}$ Nanoparticles for Efficient Removal of Cationic and Anionic Dyes. Nanomaterials 2018, 8, 162. [CrossRef] [PubMed]

9. Nassar, A.M.; Abo, Z.E.F.; Elseman, A.M.; Alotaibi, N.F. A novel heterometallic compound for design and study of electrical properties of silver nanoparticles-decorated lead compounds. New J. Chem. 2018, 42, 1387-1395. [CrossRef]

10. Ray, C.; Pal, T. Recent advances of metal-metal oxide nanocomposites and their tailored nanostructures in numerous catalytic applications. J. Mater Chem. A 2107, 5, 9465-9487. [CrossRef]

11. Liu, Y.; Kou, Q.W.; Wang, D.D.; Chen, L.; Sun, Y.T.; Lu, Z.Y.; Zhang, Y.Y.; Wang, Y.X.; Yang, J.H.; Xing, S. Rational synthesis and tailored optical and magnetic Characteristics of $\mathrm{Fe}_{3} \mathrm{O}_{4}$-Au composite nanoparticles. J. Mater. Sci. 2017, 52, 10163-10174. [CrossRef]

12. Gong, R.; Ye, J.J.; Dai, W.; Yan, X.Y.; Hu, J.; Hu, X.; Li, S.; Huang, H. Adsorptive Removal of Methyl Orange and Methylene Blue from Aqueous Solution with Finger-Citron-Residue-Based Activated Carbon. Ind. Eng. Chem. Res. 2013, 52, 14297-14303. [CrossRef]

13. Vi, T.T.T.; Kumar, S.R.; Rout, B.; Liu, C.-H.; Wong, C.-B.; Chang, C.-W.; Chen, C.-H.; Chen, D.W.; Lue, S.J.J. The Preparation of Graphene Oxide-Silver Nanocomposites: The Effect of Silver Loads on Gram-Positive and Gram-Negative Antibacterial Activities. Nanomaterials 2018, 8, 163. [CrossRef] [PubMed]

14. Du, Q.; Tan, L.; Li, B.; Liu, T.; Ren, J.; Huang, Z.; Tang, F.; Meng, X. One-pot gradient solvothermal synthesis of the $\mathrm{Ag} / \mathrm{Au}-\mathrm{Fe}_{3} \mathrm{O}_{4}$ composite nanoparticles and their applications. RSC Adv. 2014, 4, 56057-56062. [CrossRef]

15. Jin, C.J.; Han, J.; Chu, F.Y.; Wang, X.X.; Guo, R. $\mathrm{Fe}_{3} \mathrm{O}_{4} @ P A N I$ Hybrid Shell as a Multifunctional Support for Au Nanocatalysts with a Remarkably Improved Catalytic Performance. Langmuir 2017, 33, 4520-4527. [CrossRef] [PubMed]

16. Munshi, A.M.; Shi, M.; Thomas, S.P.; Saunders, M.; Spackman, M.A.; Iyer, K.S.; Smith, N.M. Magnetically recoverable $\mathrm{Fe}_{3} \mathrm{O}_{4} @ \mathrm{Au}$-coated nanoscale catalysts for the A3-coupling reaction. Dalton Trans. 2017, 46, 5133-5137. [CrossRef] [PubMed]

17. Li, W.J.; Li, D.Z.; Lin, Y.M.; Wang, P.X.; Chen, W.; Fu, X.Z.; Shao, Y. Evidence for the Active Species Involved in the Photodegradation Process of Methyl Orange on $\mathrm{TiO}_{2}$. J. Phys. Chem. C 2012, 116, 3552-3560. [CrossRef]

18. Xuan, S.H.; Wang, Y.-X.J.; Yu, J.C.; Leung, K.C.-F. Preparation, Characterization, and Catalytic Activity of Core/Shell $\mathrm{Fe}_{3} \mathrm{O}_{4} @$ Polyaniline@Au Nanocomposites. Langmuir 2009, 25, 11835-11843. [CrossRef] [PubMed]

19. Wang, Y.; Gai, L.G.; Ma, W.Y.; Jiang, H.H.; Peng, X.Q.; Zhao, L.C. Ultrasound-Assisted Catalytic Degradation of Methyl Orange with $\mathrm{Fe}_{3} \mathrm{O}_{4}$ /Polyaniline in Near Neutral Solution. Ind. Eng. Chem. Res. 2015, 54, 2279-2289. [CrossRef]

20. Xiong, R.; Lu, C.; Wang, Y.; Zhou, Z.; Zhang, X. Nanofibrillated cellulose as the support and reductant for the facile synthesis of $\mathrm{Fe}_{3} \mathrm{O}_{4} / \mathrm{Ag}$ nanocomposites with catalytic and antibacterial activity. J. Mater. Chem. A 2013, 1, 14910-14918. [CrossRef]

21. Subhan, M.A.A.; Saha, P.C.; Rahman, M.M.; Ahmed, J.; Asirib, A.M. Mohammad Al-Mamun, Fabrication of a 2,4-dinitrophenol sensor based on $\mathrm{Fe}_{3} \mathrm{O}_{4} @ \mathrm{Ag} @ \mathrm{Ni}$ nanomaterials and studies on their antibacterial properties. New J. Chem. 2018, 42, 872-881. [CrossRef] 
22. Zhang, X.P.; Jiang, W.Q.; Gong, X.L.; Zhang, Z. Sonochemical synthesis and characterization of magnetic separable $\mathrm{Fe}_{3} \mathrm{O}_{4} / \mathrm{Ag}$ composites and its catalytic properties. J. Alloys Compd. 2010, 508, 400-405. [CrossRef]

23. Liu, Y.; Jiang, Y.H.; Zhang, X.L.; Wang, Y.X.; Zhang, Y.J.; Liu, H.L.; Zhai, H.J.; Liu, Y.Q.; Yang, J.H.; Yan, Y.S. Effects of annealing temperature on the structure and magnetic properties of the $\mathrm{L}_{0}$-FePt nanoparticles synthesized by the modified sol-gel method. Powder Technol. 2013, 239, 217-222. [CrossRef]

24. Li, Q.; Wu, L.H.; Wu, G.; Su, D.; Lv, H.F.; Zhang, S.; Zhu, W.L.; Casimir, A.; Zhu, H.Y.; Mendoza-Garcia, A.; et al. New Approach to Fully Ordered fct-FePt Nanoparticles for Much Enhanced Electrocatalysis in Acid. Nano Lett. 2015, 15, 2468-2474. [CrossRef] [PubMed]

25. Hsieh, S.; Lin, P.Y. FePt nanoparticles as heterogeneous Fenton-like catalysts for hydrogen peroxide decomposition and the decolorization of methylene blue. J. Nanopart. Res. 2012, 14, 1-10. [CrossRef]

26. Wang, S.Q.; Xu, L.-P.; Zhang, X.J. Ultrasensitive Electrochemical Biosensor Based on Noble Metal Nanomaterials. Sci. Adv. Mater. 2015, 7, 2084-2102. [CrossRef]

27. Svedendahl, M.; Verre, R.; Kall, M. Refractometric biosensing based on optical phase flips in sparse and short-range-ordered nanoplasmonic layers. Light Sci. Appl. 2014, 3, e220. [CrossRef]

28. Zhu, Z.D.; Bai, B.F.; You, O.B.; Li, Q.Q.; Fan, S.S. Fano resonance boosted cascaded optical field enhancement in a plasmonic nanoparticle-in-cavity nanoantenna array and its SERS application. Light Sci. Appl. 2015, 4, e296. [CrossRef]

29. Wang, P.; Wang, Y.P.; Tong, L.M. Functionalized polymer nanofibers: A versatile platform for manipulating light at the nanoscale. Light Sci. Appl. 2013, 2, e102. [CrossRef]

30. Hunt, S.T.; Milina, M.; Alba-Rubio, A.C.; Hendon, C.H.; Dumesic, J.A.; Román-Leshkov, Y. Self-assembly of noble metal monolayers on transition metal carbide nanoparticle catalysts. Science 2016, 352, 974-978. [CrossRef] [PubMed]

31. Karabchevsky, A.; Mosayyebi, A.; Kavokin, A.V. Tuning the chemiluminescence of a luminol flow using plasmonic nanoparticles. Light Sci. Appl. 2016, 5, e16164. [CrossRef]

32. Linnenbank, H.; Grynko, Y.; Forstner, J.; Linden, S. Second harmonic generation spectroscopy on hybrid plasmonic/dielectric nanoantennas. Light Sci. Appl. 2016, 5, e16013. [CrossRef]

33. Blum, O.; Shaked, N.T. Prediction of photothermal phase signatures from arbitrary plasmonic nanoparticles and experimental verification. Light Sci. Appl. 2015, 4, e322. [CrossRef]

34. Yang, J.; Lee, J.Y.; Too, H.P. Core-Shell Ag-Au Nanoparticles from Replacement Reaction in Organic Medium. J. Phys. Chem. B 2015, 109, 19208-19212. [CrossRef] [PubMed]

35. Yang, J.H.; Jiang, Y.H.; Liu, Y.; Zhang, X.L.; Wang, Y.X.; Zhang, Y.J.; Wang, J.; Li, W.; Cheng, X. Effects of $\mathrm{SiO}_{2}$ content on the structure and magnetic properties of $\mathrm{L}_{0}$-FePt nanoparticles synthesized by the sol-gel method. Mater. Lett. 2013, 91, 348-351. [CrossRef]

36. Xing, G.Z.; Wang, Y.; Wong, J.I.; Shi, Y.M.; Huang, Z.X.; Li, S.; Yang, H.Y. Hybrid CuO/SnO 2 nanocomposites: Towards cost-effective and high performance binder free lithium ion batteries anode materials. Appl. Phys. Lett. 2014, 105, 143905-143912. [CrossRef]

37. Sun, J.H.; Sui, H.D.; Wang, Z.-W.; Wang, K.; Yan, Y.; Lu, Q.; Li, L. Preparation of europium-doped nano-TiO 2 transparent photocatalyst emulsion and photocatalytic performance. Chin. Opt. 2017, 10, 760-767. [CrossRef]

38. Xing, G.Z.; Wang, D.D.; Cheng, C.-J.; He, M.; Li, S.; Wu, T. Emergent ferromagnetism in $\mathrm{ZnO} / \mathrm{Al}_{2} \mathrm{O}_{3}$ core-shell nanowires: Towards oxide spinterfaces. Appl. Phys. Lett. 2013, 103, 022402-022407. [CrossRef]

39. Li, X.; Ji, M.; Wang, H.; Tu, G.; Wan, X.; Liu, J.; Liu, J.; Xu, M.; Zhang, J. Research progress of near-infrared photothermal conversion nanocrystals. Chin. Opt. 2017, 10, 541-554. [CrossRef]

40. Sun, Y.; Li, Q. Research of zinc oxide quantum dot light-emitting diodes based on preparation of chemical solutions. J. Liq. Cryst. Disp. 2016, 31, 635-642. [CrossRef]

41. Chen, X.Y.; Tian, Z. Recent progress in terahertz dynamic modulation based on graphene. Chin. Opt. 2017, 10, 86-97. [CrossRef]

42. Tong, L.; Zhang, M.L.; Wang, F.; Zhang, D.M.; Wang, G.P. Fabrication of optical waveguide amplifiers based on bonding-type $\mathrm{NaYF}_{4}$ : Er nanoparticles-polymer. Chin. Opt. 2017, 10, 219-225. [CrossRef]

43. Psilodimitrakopoulos, S.; Mouchliadis, L.; Paradisanos, I.; Lemonis, A.; Kioseoglou, G.; Stratakis, E. Ultrahigh-resolution nonlinear optical imaging of the armchair orientation in 2D transition metal dichalcogenides. Light Sci. Appl. 2018, 7, e18005. [CrossRef]

44. Zhang, S.; Zhang, X.; Jiang, G.M.; Zhu, H.Y.; Guo, S.J.; Su, D.; Lu, G.; Sun, S.H. Tuning nanoparticle structure and surface strain for catalysis optimization. J. Am. Chem. Soc. 2014, 136, 7734-7739. [CrossRef] [PubMed] 
45. Zhao, Y.; Tao, C.; Xiao, G.; Wei, G.; Li, L.; Liu, C.; Su, H. Controlled synthesis and photocatalysis of sea urchin-like $\mathrm{Fe}_{3} \mathrm{O}_{4} @ \mathrm{TiO}_{2} @ \mathrm{Ag}$ nanocomposites. Nanoscale 2016, 8, 5313-5326. [CrossRef] [PubMed]

46. Lu, C.Y.; Puig, T.; Obradors, X.; Ricart, S.; Ros, J. Ultra-fast microwave-assisted reverse microemulsion synthesis of $\mathrm{Fe}_{3} \mathrm{O}_{4} @ \mathrm{SiO}_{2}$ core-shell nanoparticles as a highly recyclable silver nanoparticle catalytic platform in the reduction of 4-nitroaniline. RSC Adv. 2016, 6, 88762-88769. [CrossRef]

47. Huo, J.; Zeng, H. Silver nanoparticles-sensitized cobalt complex for highly-efficient photocatalytic activity. Appl. Catal. B Environ. 2016, 199, 342-349. [CrossRef]

48. Lu, L.Y.; Wang, D.; Xu, X.G.; Wang, H.C.; Miao, J.; Jiang, Y. Low temperature magnetic hardening in self-assembled FePt/Ag core-shell nanoparticles. Mater. Chem. Phys. 2011, 129, 995-999. [CrossRef]

49. Hu, R.; Zheng, M.X.; Wu, J.C.; Li, C.; Shen, D.Q.; Yang, D.; Li, L.; Ge, M.F.; Chang, Z.M.; Dong, W.F. Core-Shell Magnetic Gold Nanoparticles for Magnetic Field-Enhanced Radio-Photothermal Therapy in Cervical Cancer. Nanomaterials 2017, 7, 111. [CrossRef] [PubMed]

50. Andreou, D.; Iordanidou, D.; Tamiolakis, I.; Armatas, G.S.; Lykakis, I.N. Reduction of Nitroarenes into

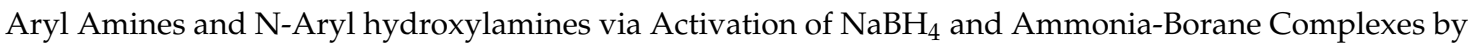
$\mathrm{Ag} / \mathrm{TiO}_{2}$ Catalyst. Nanomaterials 2016, 6, 54. [CrossRef] [PubMed]

51. Li, L.; Guo, W.; Yan, Y.; Lee, S.; Wang, T. Label-free super-resolution imaging of adenoviruses by submerged microsphere optical nanoscopy. Light Sci. Appl. 2013, 2, e104. [CrossRef]

52. Wang, D.D.; Wang, W.L.; Huang, M.Y.; Lek, A.; Lam, J.; Mai, Z.H. Failure Analysis Depa Failure mechanism analysis and process improvement on time-dependent dielectric breakdown of $\mathrm{Cu} / \mathrm{ultra}-\mathrm{low}-\mathrm{k}$ dielectric based on complementary Raman and FTIR spectroscopy study. AIP Adv. 2014, 4, 077124-077134. [CrossRef]

53. Yu, D.H.; Yu, X.; Wang, C.; Liu, X.C.; Xing, Y. Synthesis of Natural Cellulose-Templated $\mathrm{TiO}_{2} / \mathrm{Ag}_{\text {Nanosponge }}$ Composites and Photocatalytic Properties. ACS Appl. Mater. Interfaces 2012, 4, 2781-2787. [CrossRef] [PubMed]

54. Xu, L.M.; Zhang, D.D.; Ming, L.F.; Jiao, Y.C.; Chen, F. Synergistic effect of interfacial lattice $\mathrm{Ag}^{+}$and $\mathrm{Ag}^{0}$ clusters in enhancing the photocatalytic performance of $\mathrm{TiO}_{2}$. Phys. Chem. Chem. Phys. 2014, 16, 19358-19367. [CrossRef] [PubMed]

55. Wang, D.D.; Xing, G.Z.; Yan, F.; Yan, Y.S.; Li, S. Ferromagnetic (Mn, N)-codoped ZnO nanopillars array: Experimental and computational insights. Appl. Phys. Lett. 2014, 104, 022412. [CrossRef]

56. Pincella, F.; Isozaki, K.; Miki, K. A visible light-driven plasmonic photocatalyst. Light Sci. Appl. 2014, 3, 110-118. [CrossRef]

57. Xing, G.Z.; Fang, X.S.; Zhang, Z.; Wang, D.D.; Huang, X.; Guo, J.; Liao, L.; Zheng, Z.; Xu, H.R.; Yu, T. Ultrathin single-crystal ZnO nanobelts: Ag-catalyzed growth and field emission property. Nanotechnology 2010, 21, 255701-255710. [CrossRef] [PubMed]

58. Wang, B.; Qu, S. Absorption spectra and near-electric field enhancement effects of $\mathrm{Au}$ - and $\mathrm{Ag}-\mathrm{Fe}_{3} \mathrm{O}_{4}$ dimers. Appl. Surf. Sci. 2014, 292, 1002-1008. [CrossRef]

59. Zhang, X.; Song, L.; Cai, L.; Tian, X.; Zhang, Q.; Qi, X.; Zhou, W.; Zhang, N.; Yang, F.; Fan, Q.X.; et al. Optical visualization and polarized light absorption of the single-wall carbon nanotube to verify intrinsic thermal applications. Light Sci. Appl. 2015, 4, e318. [CrossRef]

60. Xing, G.Z.; Yi, J.B.; Wang, D.D.; Liao, L.; Yu, T.; Shen, Z.X.; Huan, C.H.A.; Sum, T.C.; Ding, J.; Wu, T. Strong correlation between ferromagnetism and oxygen deficiency in Cr-doped $\operatorname{In}_{2} \mathrm{O}_{3-\delta}$ nanostructures. Phys. Rev. B 2009, 79, 174406-174415. [CrossRef]

61. Xing, G.Z.; Yi, J.B.; WU, T. Comparative Study of Room-Temperature Ferromagnetism in Cu-Doped ZnO Nanowires Enhanced by Structural Inhomogeneity. Adv. Mater. 2008, 20, 3521-3527. [CrossRef]

62. Zhang, X.; Niu, C.; Wang, Y.; Zhou, S.; Liu, J. Gel-limited synthesis of dumbbell-like $\mathrm{Fe}_{3} \mathrm{O}_{4}$-Ag composite microspheres and their SERS applications. Nanoscale 2014, 6, 12618-12625. [CrossRef] [PubMed]

63. Wang, J.M.; Wang, Z.L.; Li, S.; Wang, R.M.; Song, Y.J. Surface and interface engineering of FePt/C nanocatalysts for electro-catalytic methanol oxidation: Enhanced activity and durability. Nanoscale 2017, 9, 4066-4075. [CrossRef] [PubMed]

64. Krylova, G.; Giovanetti, L.J.; Requejo, F.G.; Dimitrijevic, N.M.; Prakapenka, A.; Shevchenko, E.V. Study of Nucleation and Growth Mechanism of the Metallic Nanodumbbells. J. Am. Chem. Soc. 2012, 134, 4384-4392. [CrossRef] [PubMed] 
65. Niedermaier, I.; Kolbeck, C.; Steinrück, H.P.; Maier, F. Dual analyzer system for surface analysis dedicated for angle-resolved photoelectron spectroscopy at liquid surfaces and interfaces. Rev. Sci. Instrum. 2016, 87, 045105. [CrossRef] [PubMed]

66. Sahu, N.K.; Bahadur, D. Influence of excess Fe accumulation over the surface of FePt nanoparticles: Structural and magnetic properties. J. Appl. Phys. 2013, 113, 134303-134313. [CrossRef]

67. Goon, I.Y.; Lai, L.M.H.; Lim, M.; Munroe, P.; Gooding, J.J.; Amal, R. Fabrication and Dispersion of Gold-Shell-Protected Magnetite Nanoparticles: Systematic Control Using Polyethyleneimine. Chem. Mater. 2009, 21, 673-681. [CrossRef]

68. Wang, Y.; Shen, Y.; Xie, A.; Li, S.; Wang, X.; Cai, Y. A Simple Method To Construct Bifunctional Fe $\mathrm{O}_{4} / \mathrm{Au}$ Hybrid Nanostructures and Tune Their Optical Properties in the Near-Infrared Region. J. Phys. Chem. C 2010, 114, 4297-4301. [CrossRef]

69. Lopes, G.; Vargas, J.M.; Sharma, S.K.; Beron, F.; Pirota, K.R.; Knobel, M.; Rettori, C.; Zysler, R.D. Ag-Fe $\mathrm{O}_{4} \mathrm{O}_{4}$ Dimer Colloidal Nanoparticles: Synthesis and Enhancement of Magnetic Properties. J. Phys. Chem. C 2010, 114, 10148-10152. [CrossRef]

70. Freitas, M.; Sá, C.M.; Barroso, M.F.; Pereira, C.; De-Los-Santos-Álvarez, N.; Miranda-Ordieres, A.J.; Lobo-Castañón, M.J.; Delerue-Matos, C. Highly Monodisperse $\mathrm{Fe}_{3} \mathrm{O}_{4} @ \mathrm{Au}$ Superparamagnetic Nanoparticles as Reproducible Platform for Genosensing Genetically Modified Organisms. ACS Sens. 2016, 1, 1044-1053. [CrossRef]

71. Lu, S.X.; Yu, J.Y.; Cheng, Y.Y.; Wang, Q.; Barras, A.; Xu, W.G.; Szunerits, S.; Cornu, D.; Boukherroub, R. Preparation of silver nanoparticles/polydopamine functionalized polyacrylonitrile fiber paper and its catalytic activity for the reduction 4-nitrophenol. Appl. Surf. Sci. 2017, 411, 163-169. [CrossRef]

72. Zhang, K.H.; Wang, C.W.; Rong, Z.; Xiao, R.; Zhou, Z.; Wang, S.Q. Silver coated magnetic microflowers as efficient and recyclable catalysts for catalytic reduction. New J. Chem. 2017, 41, 14199-14208. [CrossRef]

73. Khan, M.M.; Lee, J.; Cho, M.H. Au@TiO 2 nanocomposites for the catalytic degradation of methyl orange and methylene blue: An electron relay effect. J. Ind. Eng. Chem. 2014, 20, 1584-1590. [CrossRef]

74. Wysocka, I.; Kowalska, E.; Trzcinski, K.; Łapinski, M.; Nowaczyk, G.; Zielinska-Jurek, A. UV-Vis-Induced Degradation of Phenol over Magnetic Photocatalysts Modified with Pt, Pd, Cu and Au Nanoparticles. Nanomaterials 2018, 2, 28. [CrossRef] [PubMed]

75. Li, M.; Li, X.G.; Qi, X.H.; Luo, F.; He, G.H. Shape-Controlled Synthesis of Magnetic Iron Oxide@SiO $2-\mathrm{Au} @ \mathrm{C}$ Particles with Core-Shell Nanostructures. Langmuir 2015, 31, 5190-5197. [CrossRef] [PubMed] 